\title{
Establishment of a Technological Platform for Pre-Clinical Evaluation of Biomedical Cellular Products in Russia
}

\author{
P.I. Makarevich, Yu P. Rubtsov, D.V. Stambolsky, N.I. Kalinina, \\ Zh A. Akopyan, Y.V. Parfyonova and V.A. Tkachuk
}

\begin{abstract}
After joint legislative efforts of scientific community and Government resulted in the adoption of Federal Law \#180 "On biomedical cellular products (BMCP)" onset of first pre-clinical trials of cell therapies in Russia got into scope. Testing of BMCPs to assess their safety and obtain primary efficacy results is a cornerstone of development and translation to clinical trials. Thus, a task force consisting of Lomonosov Moscow State University, leading research groups and experts from both - regulatory and industrial entities have been established under a project funded by Russian Ministry of Science and Education. As far as Federal Law \#180 is enforced starting January 1, 2017, completion of the project in December 2016 is a timely step to ensure the development of cell therapies and regenerative medicine in Russia. The present article gives an overview of the project in 2014-2016 and summarizes main results of the collaborative effort.
\end{abstract}

Keywords Federal law \#180 - Biomedical cellular products - Tissue regeneration Cell transplantation - Biosafety - Manufacture - Evaluation - Pre-clinical testing Cell manipulation - Bioproduct storage and transportation - Cryoconservation Quality control - Mesenchymal stem cells • Progenitors of cardiac stem cells Platelet lysate $\cdot$ Genetically modified cells

\section{Introduction}

Modern biomedicine has reached a point when new revolutionary approaches based on the concept of regenerative medicine allow treatment of human disease not alleviated by existing drugs or surgical technique. Regenerative medicine employs the concept of the natural process of tissue/organ repair and renewal driven by specific cellular elements_-namely stem and progenitor cells. Cultural approaches

P.I. Makarevich $(\bowtie)$ · Y.P. Rubtsov · D.V. Stambolsky · N.I. Kalinina · Z.A. Akopyan Y.V. Parfyonova · V.A. Tkachuk

Lomonosov Moscow State University, Moscow, Russia

e-mail: pmakarevich@mc.msc.ru 
advanced rapidly and now allow to grow human cells in vitro, generate specific cell types using induced pluripotency protocol, create artificial organs/tissues, and to correct mutations in cells by genome editing (mediated by CRISPR/Cas9 system). Furthermore, in addition to systemic transplantation, there are multiple options to introduce therapeutic cells to patient's organism; these include combining cells with extracellular matrix, synthetic scaffolds or cell-derived secreted products to ensure engraftment and/or provide structural support where required (e.g., nerve repair, muscle defect filling or vascular repair).

However, unique properties of BCMP come along with major safety concerns including the risk of carcino-and tumorogenesis, ectopic tissue outgrowth, immunotoxic effects, and adverse reactions related to transplant rejection. Besides serious adverse reactions, limited knowledge of BMCP kinetics (survival and distribution) and pharmacology (including secondary and off-target effects) is another point that requires thorough analysis prior to first-in-human use. European and USA regulatory institutes have established a series of legal acts to control the field of cell therapy and increase its positive impact with reduction of potential harm $[1$, p. 2; 4, p. 2; 5, p. 2].

Initiation of clinical trials is crucial to advance in the field and develop efficient and safe BMCP to cure previously moribund and lethal conditions. However, abovementioned reasons clearly show that due to natural and scientific reasons evaluation of their safety and efficiency are challenging and requires efforts from both scientific community and regulatory entities. Emerging cell-and cell-based product medical technologies are novel for Russian Federation, therefore, prior to the introduction of Federal Law \#180, the Ministry of Science and Education had initiated a complex project to establish a "technological platform" and develop reasonable regulation for procedures of pre-clinical testing of BMCP [2, p. 2].

\section{Legal Field, Workgroup, and Project Goal}

Until recent no specific "stem cell law" has existed in Russian Federation to regulate development, testing, manufacture, registration, and marketing of cell-based therapeutic products. Indeed, use of human cells (with exception of blood transfusion and organ transplantation) in the clinic has remained a "gray area" where successes of highly professional scientific groups were formally adjacent to procedures conducted by commercial clinics which offered cell-based therapy for various conditions. Lack of regulation led to use of poorly characterized cells without proper monitoring and control of results.

Federal Law \#180 has been signed by the President of Russian Federation and will be enforced since January 1, 2017 to regulate all aspects of cell therapy in Russian Federation. This law regulates design, manufacture, quality and safety evaluation, transportation, clinical trial initiation and marketing of BMCP. These products consist or contain human cells and may contain modified human cells (not organs or human cells used for blood transfusion) obtained from human donors, 
which can be used for treatment by itself or in combination with other drugs or materials. Generally, BMCP is a new class of medicinal products covering the niche between drugs and transplanted organs/tissues. From one side BMCP has defined active components responsible for therapeutic efficiency, and, from the other side, they consist of cells which produce active components inside the body in a more physiological manner and interact with surrounding cells at the site of injection/introduction. The law defines major terms and limitations of BMCP production, handling, testing, and use.

Major issues covered by the law are presented below:

(1) Prohibition of embryonic material (including cloned embryos) use to generate $\mathrm{BMCP}$ and/or cell lines for BMCP production; the point arises from two main reasons: ethical concerns associated with use of abortive material and safety issues related to teratogenic potential of embryonic cells;

(2) Definition of BMCP classes includes autologous, allogeneic, genetically modified and combined - the latter are BMCPs containing both-cells and acellular component(s) such as drugs or medical devices;

(3) Requirements for BMCP production such as criteria for donor material, testing, handling, transportation, and evaluation of final product; organizations and companies manufacturing BMCP must comply with GMP standards for production of BMCP for clinical use;

(4) Procedures for BCMP registration and authorization for clinical trials of BMCP is stipulated; expertise of pre-clinical data is stated as a crucial point for first-in-human use and aggregated data of data on ethical, quality control and efficacy data are required for marketing of BMCP.

Project's main goal lies within the scope of the newly accepted Federal Law and arising demand for legal action to ensure effective pre-clinical testing of BMCP. It can be sub-divided into three major tasks:

(1) Establish a technological platform for pre-clinical evaluation of BMCPs;

(2) Propose and draft guidelines for conduct of pre-clinical evaluation of BMCP;

(3) Prepare programs and modules required for professional education of specialists in development, manufacture, and pre-clinical evaluation of BMCP

A consortium, consisting of Lomonosov Moscow State University, Pirogov Russian National Research Medical University, Gertsen Moscow Research Oncology Institute, Almazov Federal Medical Research Center, Russian Cardiology Research and Production Complex, Shumakov Federal Centre of Transplantation and Artificial Organs, Central Institution of Tuberculosis and Scientific Centre for Expertise of Medical Products was formed to develop the project. As a starting point, besides in-house developments of each organization we used the international experience as a reference, and based on that tried to develop practical recommendations for design and pre-clinical evaluation of certain types of BMCP. 
Thus, the new law defines BMCPs, and how they are to be generated, tested, and used. It sets ethical, commercial, and technical limitations of the BMCP testing, production, and practical use. But these are mostly common words related to legislative part of the problem. In reality, there are some fundamental problems concerning testing of BMCP's safety and mechanisms of action. First of all, by definition BMCPs are human cell lines, which have to be biologically tested prior to clinical trials. This is not so simple since in most animal models transferred human cells will be immunologically rejected. Another evident problem is that it is hard to use the pre-clinical data obtained, for example, in small rodents to calculate numbers of cells that have to be used in humans due to physiological reasons. And there are many more examples of challenges of pre-clinical testing of BMCPs. Therefore, a need in the development of experimental protocols and recommendations regarding pre-clinical testing of BMCP exists.

\section{Project Industrial Partner}

CellThera Pharm is a pioneer national project realized by the Russian pharmaceutical company "Pharmstandard" incorporated on September 2013 is a full-cycle regenerative medicine company. At the disposal of the company has an R\&D department, animal facility and a modern manufacturing facility for development and production of advanced medicinal products based on cell technology.

CellThera Pharm develops the products for unmet medical needs with innovative approaches based on scientific excellence to provide the Russian Federation public healthcare system with fully personalized modern advanced medicinal products manufactured locally for the treatment of severe and socially significant diseases:

- Personalized immunotherapy for cancer treatment

- Aesthetic surgery/reconstructive surgery

To date in the disposition of CellThera Pharm are following facilities with a total area of $>900 \mathrm{~m}^{2}$ including:

- manufacturing site, based on the isolator technology

- R\&D department

- animal facility

The core competencies of Cellthera Pharm are: Cell biology, immunology, Cells and tissue technologies, Molecular biology, Tech-transfer, and technology optimization, pre-clinical study and strong expertise in animal model development

The Cellthera's development strategy provides intensive development and consists both in the development of innovative products and the licensing of the best technologies that are currently present in the world. 


\section{Key Results and Product Description}

The project's main goal was to develop a technological platform for pre-clinical evaluation of biomedical cell products. A consortium of 8 medical and scientific centers headed by Lomonosov Moscow State University accomplished this mission successfully in 2.5 years with regular reports to the Ministry. Overall, the project wraps up with a total of 8 patents pending, 5 papers in Scopus/WoS indexed journals and 6 conference proceedings to popularize and spread the results and current progress.

Member-organizations of the consortium used their scientific expertise and development experience conducting experiments required to elaborate parameters for BMCP evaluation of safety, efficacy, and distribution. Furthermore, a significant part of the project was devoted to the establishment of appropriate standards for BMCP manufacture and required quality control - these results were formulated as in-house manufacture regulations and standard operation procedures (SOPs).

BMCP from different cell types were evaluated: mesenchymal stem cells from adipose tissue and bone marrow (AD MSC and BM MSC, respectively), progenitors of cardiomyocytes (PC), and bio product based on platelet lysate (BPPL). Analysis of literature allowed the definition of criteria crucial for design, manufacturing, evaluation, in vitro and in vivo pre-clinical testing of each biomedical product. According to these criteria members of consortium thoroughly selected and tried various experimental protocols to determine the clinical efficiency of BMCP in animal models of human pathological conditions. Another set of experiments was used to evaluate stability, distribution in a body, and interaction of BMCP with surrounding tissue. The third line of tests was conducted to prove the biological safety of BMCP.

Laboratory protocols suitable for manufacturing of different BMCPs were generated. Different BMCPs were evaluated for efficiency in animal models. For example, BMCP based on AD MSC was tested in the model of myocardial infarction, and BMCP based on BM MSC - in the model of acute graft versus host disease caused by organ transplantation. AD MSC-mediated immune suppression was studied in the course of tuberculosis infection in mice. BMCPs derived from human MSC were studied for the ability to facilitate regeneration of joint cartilage, liver, and pancreas in rodents. BMCP from human PC passed tests for angio-, arteriogenesis, the effect on fibrosis, cardiomyocyte proliferation, inflammatory infiltration, and myocardium remodeling in a model of rat myocardium injury. BPPL influence on cultures of MSC and fibroblasts, as well as biosafety, was studied. Another BMCP designed for use on damaged skin and possessing dermatotropic properties were tested in mouse skin repair model.

Multiple biosafety tests and protocols were designed for abovementioned BMCP. As a result, now we have a collection of standard protocols and practical recommendation which will simplify life for those who will design, characterize, manufacture, and evaluate BMCP in Russia in the future.

The overall goal of the project was achieved by joint effort and draft of practical recommendations (Guidelines) for design and conduct of pre-clinical evaluation of 
BMCP in general and particular examples of BMCPs. These Guidelines contain scientifically reasoned non-binding recommendations for evaluation of BMCPs pharmacology, kinetics (terms used on basis of lack of a suitable alternative) and safety including toxicity and secondary pharmacology. Much attention was paid to evaluation using homologous animal cell cultures in appropriate animal models required for certain situations of BMCP evaluation, especially in immune-modulating therapies.

Moreover, Guidelines were drafted as a partially harmonized regulation with internationally adopted regulatory documents and experience of FDA-and EMA-approved cell therapeutic drugs was taken into account during development.

Another important objective achieved was the development of educational programs for physicians and specialists in pre-clinical evaluation of BMCPs drafted to ensure proper standards for professionals. These programs will be required after enforcement of Federal Law \#180 to propagate and educate healthcare workers and researchers endeavoring stem cell therapies as their primary field of work.

Results of the project will be in demand by the Industrial partner and whole field of regenerative medicine ranging from developers and researchers to regulatory authorities and federal government. Developed guidelines after approval will be used by the researchers, government-based and contract organizations to plan pre-clinical testing of developed BMCPs and fully evaluate their safety and biological properties crucial for efficacy in clinical settings.

\section{Conclusions}

It should be noted that despite BMCPs are used for treatment and can be to a certain extent compared with commonly used chemical-based drugs it has a lot of differences from low-molecular weight or antibodies used as therapeutic agents. BMCPs carry living cells and thus cannot be sterilized and have minimal (hours long) shelf-life. Furthermore, immune response to cellular antigens may induce toxicity and transplant rejection, thus, additional requirements for donor compatibility or autologous approach are enforced.

Complex nature of BMCP implies that pre-clinical testing one hand has to be in general framework of safety assessment and on the other has to take into account specific indications, routes of delivery and specific features of cells use for BMCP manufacture [p. 7, 3]. Thus, additional testing is to be applied to control kinetics (distribution and fate of delivered cells) and pharmacological effects. Adequate animal models are also an issue as far as some human-based BMCPs are immunogenic in other mammals and, thus, immunosuppressed or immunodeficient transgenic animal strains are to be used for testing [p. 7, 6].

Thus, to conclude, our project was a very timely attempt to establish a reasonable regulation of pre-clinical assessment for newly developed biomedical products relying on the potency of human cells as a "drug." We managed to create a methodology for the harvesting of donor cells, obtaining of cell cultures, maintenance, and storage of biomaterials, cytological and biochemical characterization of 
final products, biosafety and efficacy assessment. On this basis we stipulated another crucial point and drafted the general Guidelines that can be applied for BMCPs yet may require a certain extent of interaction with regulatory authorities to ensure certain clarity in ambiguous situations. Further challenges in the field are numerous ranging from marketing to clinical trial organizations and the scientific community is ready for further cooperation with federal government and industry to use its expertise for the sake of the progress of the biomedical field.

Acknowledgment Research being carried out with the financial support of the state represented by the Ministry of Education and Science of the Russian Federation. Contract no. 14.610.21.0001 03 Oct. 2014. Unique project Identifier: RFMEFI61014X0001.

\section{References}

1. Directive 2001/20/EC of the European parliament and of the council of 4 April 2001. Online resource: http://ec.europa.eu/health/human-use/clinical-trials/directive/index_en.htm. Accessed 27 Nov 2016)

2. Federal Law of Russian Federation: On biomedical cellular products. 23.06.2016 N180-FZ (current edition 27.11.2016)

3. Guidance for Industry: Preclinical Assessment of Investigational Cellular and Gene Therapy Products. Online resource: http://www.fda.gov/BiologicsBloodVaccines/GuidanceCompliance RegulatoryInformation/Guidances/CellularandGeneTherapy/ucm376136.htm. Accessed 26 Nov 2016

4. Guidance for Industry: Regulation of Human Cells, Tissues, and Cellular and Tissue-Based Products (HCT/Ps). Online resource: http://www.fda.gov/downloads/BiologicsBloodVaccines/ GuidanceComplianceRegulatoryInformation/Guidances/Tissue/ucm062592.pdf. Accessed 28 Nov 2016

5. Regulation (EC): No 1394/2007 of the European Parliament and of the Council of 13 November 2007 on advanced therapy medicinal products. Online resource: https://www. eumonitor.eu/9353000/1/j9vvik7m1c3gyxp/vi7jgtb6qkzs. Accessed 28 Nov 2016

6. USA CFR 21, part 1271 "Human cells, tissues, and cellular and tissue-based products» . Online resource: http://www.accessdata.fda.gov/scripts/cdrh/cfdocs/cfcfr/CFRSearch.cfm? CFRPart=1271. Accessed 27 Nov 2016

Open Access This chapter is licensed under the terms of the Creative Commons Attribution 4.0 International License (http://creativecommons.org/licenses/by/4.0/), which permits use, sharing, adaptation, distribution and reproduction in any medium or format, as long as you give appropriate credit to the original author(s) and the source, provide a link to the Creative Commons license and indicate if changes were made.

The images or other third party material in this chapter are included in the chapter's Creative Commons license, unless indicated otherwise in a credit line to the material. If material is not included in the chapter's Creative Commons license and your intended use is not permitted by statutory regulation or exceeds the permitted use, you will need to obtain permission directly from the copyright holder. 